\title{
Bivariate linkage analysis of cholesterol and triglyceride levels in the Framingham Heart Study Xuyang Zhang ${ }^{1}$ and Kai Wang*2
}

Address: ${ }^{1}$ Department of Speech Pathology and Audiology, The University of Iowa, Iowa City, USA and ${ }^{2}$ Department of Biostatistics, Division of Statistical Genetics, The University of Iowa, Iowa City, Iowa, USA

Email: Xuyang Zhang - xuyang-zhang@uiowa.edu; Kai Wang* - kai-wang@uiowa.edu

* Corresponding author

from Genetic Analysis Workshop I3: Analysis of Longitudinal Family Data for Complex Diseases and Related Risk Factors New Orleans Marriott Hotel, New Orleans, LA, USA, November I I-I4, 2002

Published: 3I December 2003

BMC Genetics 2003, 4(Suppl I):S62

This article is available from: http://www.biomedcentral.com/I47I-2I56/4/sI/S62

\begin{abstract}
We performed a bivariate analysis on cholesterol and triglyceride levels on data from the Framingham Heart Study using a new score statistic developed for the detection of potential pleiotropic, or cluster, genes. Univariate score statistics were also computed for each trait. At a significance level $0.00 \mathrm{I}$, linkage signals were found at markers GATA48B0I on chromosome I, GATA2ICI 2 on chromosome 8, and ATA55AII on chromosome 16 using the bivariate analysis. At the same significance level, linkage signals were found at markers $036 y b 8$ on chromosome 3 and GATA3F02 on chromosome 12 using the univariate analysis. A strong linkage signal was also found at marker GATAII2F07 by both the bivariate analysis and the univariate analysis, a marker for which evidence for linkage had been reported previously in a related study.
\end{abstract}

\section{Background}

Elevated triglyceride and cholesterol levels are two risk factors for cardiovascular diseases. These risk factors are often correlated with each other. In order to map the possible pleiotropic/clustered genes underlying the inheritance of these two traits, we performed a bivariate linkage analysis using a score statistic developed by Wang [1]. This score statistic is asymptotically equivalent to the likelihood ratio statistic and is straightforward to compute. We apply this statistic to data from Cohort 1 and Cohort 2 of the Framingham Heart Study.

\section{Methods}

Data

Participants in Cohort 1 had up to 16 reported cholesterol levels, and up to 3 reported triglyceride levels. For participants in Cohort 2, cholesterol and triglyceride levels were reported up to 5 times. These two cohorts together provided 22,040 measurements on the cholesterol level and
9,155 measurements on the triglyceride level (including all repeated measurements on all individuals). Individuals who lacked any measurements of cholesterol level or triglyceride level were excluded. A single linear regression of cholesterol on age was fit across different individuals and different measurements. The residuals from the regression fit were averaged for each individual. This average was used as the age-adjusted cholesterol level for that individual. The same method was used to obtain ageadjusted triglyceride level for each individual. Sib pairs from the same nuclear family or from different nuclear families that belonged to the same pedigree were regarded as biologically unrelated. For the case of univariate traits, there are reports showing that treating dependent sib pairs as independent ones does not increase the type I error rate of the test [2].

All sib pairs in all the pedigrees in Cohort 1 and Cohort 2 were generated, but not all of these sib pairs were used at 
Table I: Number of markers available and analyzed on each chromosome

\begin{tabular}{lccc}
\hline Chr & Markers Provided & Markers Analyzed & Sib Pairs \\
\hline 1 & 32 & 30 & 965 \\
2 & 30 & 28 & 981 \\
3 & 27 & 27 & 380 \\
4 & 21 & 21 & 1215 \\
5 & 26 & 24 & 719 \\
6 & 23 & 19 & 1187 \\
7 & 22 & 20 & 1245 \\
8 & 19 & 19 & 1298 \\
9 & 19 & 401 & 970 \\
10 & 22 & 17 & 788 \\
11 & 17 & 20 & 1178 \\
12 & 19 & 15 & 1524 \\
13 & 12 & 18 & 1292 \\
14 & 17 & 11 & 1318 \\
15 & 13 & 14 & 1218 \\
16 & 14 & 12 & 1284 \\
17 & 16 & 14 & 1426 \\
18 & 15 & 15 & 1715 \\
19 & 11 & 14 & 1843 \\
21 & 11 & 10 & 10 \\
22 & 6 & 6 & \\
\hline
\end{tabular}

AThe sample size is the same for all markers on the same chromosome.

the same time due to missing marker data. Genetic Analysis Workshop 13 (GAW13) provided identity-by-descent (IBD) sharing probabilities for some relative pairs (including sib pairs) at each of the scanned markers. The IBD sharing probabilities for a sib pair were available only for some markers. To simplify the programming, we excluded those markers at which there were less than 1000 sib pairs whose IBD sharing probabilities were available. Then, for each chromosome, we used only those sib pairs whose IBD sharing probabilities were available for all the remaining markers on that chromosome. See Table 1 for a summary of the number of markers excluded and the number of sib pairs used for each chromosome.

\section{Analysis}

The bivariate score statistic is computed based on the observed phenotypic data on sib pairs. The phenotypic data of a sib pair can be denoted by a vector of four (adjusted) measurements - cholesterol levels on sib 1 and sib 2, and triglyceride levels on sib 1 and sib 2 . Let $x_{i}$ be the phenotypic data on the $i^{\text {th }}$ sib pair and $\Sigma_{0}$ be the sample variance-covariance of $x_{i}$. As an average of the residuals of a regression, the sample mean of cholesterol levels on sib 1 and sib 2 is 0 , so is the sample mean of triglyceride level. Let $\Sigma_{0}$ be a $4 \times 4$ symmetric matrix whose $(i, j)$ element is denoted by aij. Note that $\mathrm{a}_{11}$ and $\mathrm{a}_{33}$ are the variances of the cholesterol and triglyceride levels, respectively, of the first sib in the pairs. Similarly, $\mathrm{a}_{22}$ and $\mathrm{a}_{44}$ are the variances of the cholesterol and triglyceride levels of the second sib in the sib pairs. The off-diagonal terms represent covariances: $a_{13}=a_{31}$ is the covariance between cholesterol and triglycerides for the first sib in the sib pairs, and $\mathrm{a}_{24}=\mathrm{a}_{42}$ is the covariance for the second sib in the sib pairs. Since the sib-sib relationship in a sib pair is symmetric, we expect that $\mathrm{a}_{11} \approx \mathrm{a}_{22}, \mathrm{a}_{33} \approx \mathrm{a}_{44}$ and $\mathrm{a}_{13} \approx \mathrm{a}_{24}$ when the sample size is large. Alternatively, we can also use the (adjusted) measurements on cholesterol and triglycerides on all sibs (do not distinguish sib 1 from sib 2) in calculating the entries of $\Sigma_{0}$. Then there would be $\mathrm{a}_{11}=$ $a_{22}, a_{33}=a_{44}$, and $a_{13}=a_{24}$. Since the sample size is fairly large, we expect both methods give similar $\Sigma_{0}$.

Define

$\mathbf{w}_{\mathbf{i}}=\left(w_{\mathrm{i} 1}, w_{\mathrm{i} 2}, w_{\mathrm{i} 3}, w_{\mathrm{i} 4}\right)^{\mathrm{t}}=\sum_{0}^{-1} \mathbf{x}_{\mathbf{i}}$

and

$z_{\mathrm{i}}=w_{\mathrm{i} 1} w_{\mathrm{i} 2} a_{11}+\left(w_{\mathrm{i} 1} w_{\mathrm{i} 3}+w_{\mathrm{i} 2} w_{\mathrm{i} 4}\right) a_{13}+w_{\mathrm{i} 3} w_{\mathrm{i} 4} a_{33}$.

Denote the proportion of alleles that are shared IBD by the $i^{\text {th }}$ sib pair by $\pi_{\mathrm{i}}$. Let $\bar{\pi}$ and $\bar{z}$ be the sample means of $\left\{\pi_{\mathrm{i}}\right\}$ and $\left\{\mathrm{z}_{\mathrm{i}}\right\}$, respectively. Define 
$b=\sum_{i=1}^{N}\left(\pi_{i}-\bar{\pi}\right)\left(z_{i}-\bar{z}\right)$,

where $N$ is the total number of sib pairs. When the putative locus is not linked to any trait locus, the expectation of $b$ is 0 and its variance is $\operatorname{Var}(\mathrm{b})=\mathrm{N} \mathrm{s}^{2}{ }_{\pi} \mathrm{s}^{2}{ }_{\mathrm{z}^{\prime}}$, where $\mathrm{s}^{2}{ }_{\pi}$ and $\mathrm{s}^{2}{ }_{\mathrm{z}}$ are the sample variances of $\left\{\pi_{\mathrm{i}}\right\}$ and $\left\{\mathrm{z}_{\mathrm{i}}\right\}$, respectively. The score statistic $S$ for the bivariate phenotypes is defined by $S=b^{2} / \operatorname{Var}(b)$ if $b>0 ; S=0$ otherwise. When the putative locus is not linked to any quantitative trait loci (QTL), the asymptotic distribution of this one-sided tests statistic, $S$, is $0.5 \chi^{2}{ }_{0}+0.5 \chi^{2}{ }_{1}[1]$. The score statistic $S$ is a special case described by Wang [1] - the locus specific variances and covariance for the two traits are assumed to be proportional to their total variances and covariance.

\section{Results}

The score statistic $S$ was calculated for every screened marker. In addition, the univariate score statistic of Wang and Huang [3] was also calculated for cholesterol level and triglyceride level separately. For sib-pair data, the type of data used in our analyses, this univariate score statistic is equivalent to other methods [4,5]. The $p$-values of these three score statistics (one for the bivariate phenotypes, one for each of the two univariate phenotypes) at each marker location are plotted in Figure 1. Markers with $p$ values less than the significance level of $\alpha=0.005$ are shown in Table 2.

At the significance level 0.005, 10 markers were identified by the bivariate score statistic: 2 each from chromosome 1 (at $212 \mathrm{cM}$ and $233 \mathrm{cM}$ ) and 7 (at $128 \mathrm{cM}$ and $155 \mathrm{cM}$ ), and 1 each from chromosome 3 (at $112 \mathrm{cM}$ ), 4 (at 105 cM), 5 (at $19 \mathrm{cM}$ ), 6 (at $166 \mathrm{cM}$ ), 8 (at $140 \mathrm{cM}$ ), and 16 (at $64 \mathrm{cM}$ ). Five out of the 10 markers were also identified by the univariate score statistic for the adjusted triglyceride level. They were the two on chromosome 1, one on chromosome 7 (at $155 \mathrm{cM}$ ), one on chromosome 8 , and one on chromosome 16 . None of the 10 markers were identified by the univariate score statistic for the ageadjusted cholesterol level. The results seem to suggest that there were large overlaps of linkage signals between the bivariate score statistic and the univariate score statistic for the age-adjusted triglyceride level. There were no overlaps of linkage signals between the bivariate score statistic and the univariate score statistic for the age-adjusted cholesterol level. There were 5 markers that were identified by the bivariate score statistic, but not identified by any of the univariate score statistics. There were 3 markers whose $p$ values were below 0.001: one on chromosome 1 at 212 $\mathrm{cM}$, one on chromosome 8 at $140 \mathrm{cM}$, and the other on chromosome 16 at $64 \mathrm{cM}$. The regions suggested by these 3 markers may be investigated in future genotyping and analysis.

\section{Discussion}

We performed a bivariate analysis of cholesterol and triglyceride levels on sib-pair data from the Framingham Heart Study using a method recently developed by Wang [1]. This method is asymptotically equivalent to the likelihood ratio statistic, but is straightforward to calculate. We also calculated the univariate score statistics for cholesterol and triglyceride levels separately. Five markers were identified by both the bivariate score statistic and the univariate score statistic for the adjusted triglyceride level, while the results of the bivariate score statistics had no overlap with the univariate score statistic for the age adjusted cholesterol levels.

The method in Wang [1] is general enough to handle general pedigrees, but we only applied it to sib pairs that were extracted from general pedigrees. This is because the programming for sib pairs is relatively easy and was feasible given the time constraint for GAW13. Some linkage information may have lost due to the fact that dependent sib pairs were treated as independent sib pairs, but the type I error rate of the test statistic is expected to be valid.

In a related study, Shearman et al. [6] used the ratio of triglyceride level to high-density lipoprotein cholesterol level as the phenotype of interest. Linkage evidence was reported at marker GATA112F07 (155 cM on chromosome 7 ), a marker that resulted in a $p$-value 0.0020 for the bivariate score statistic used in the current report. These authors reported a LOD score 1.5 at $70 \mathrm{cM}$ on chromosome 16 with multipoint mapping. We used single-point IBD sharing probabilities with the bivariate score statistic and obtained a significant linkage signal $(p=0.0001)$ for marker ATA55A11 (64 cM on chromosome 16), $6 \mathrm{cM}$ away from the locus they identified. Other markers in Table 2 that have small $p$-values for the bivariate or univariate score statistics but that did not show evidence for linkage in Shearman et al. [6] include GATA48B01, 036yb8, GATA21C12, and GATA3F02.

One caveat about bivariate analyses is that they are not always more powerful than univariate analyses. Theoretical [7] and simulation studies [1,8,9] demonstrate that when the polygenic correlation is in the same direction as the major gene correlation, a bivariate analysis may have lower power than a univariate analysis. 
A

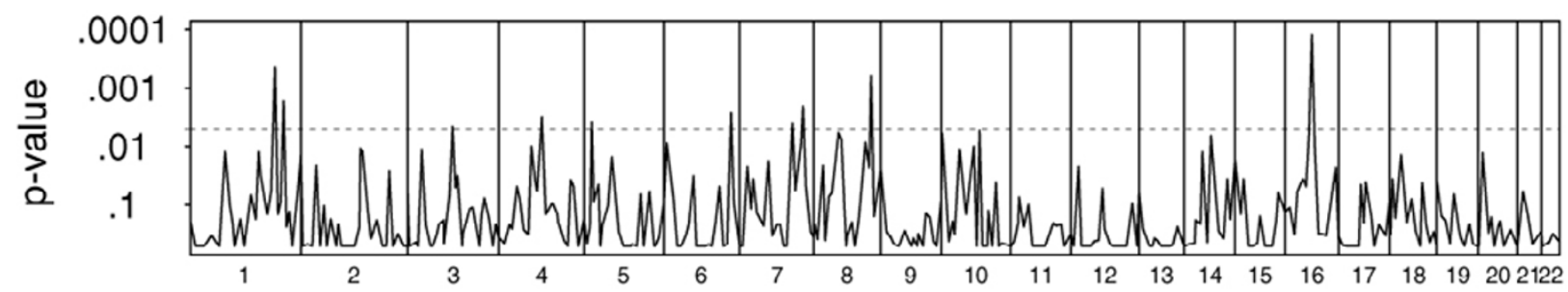

B

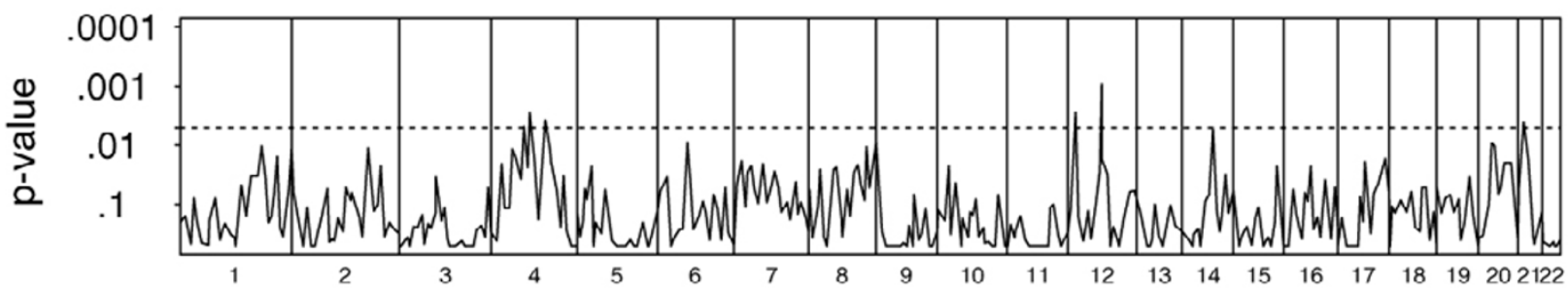

C

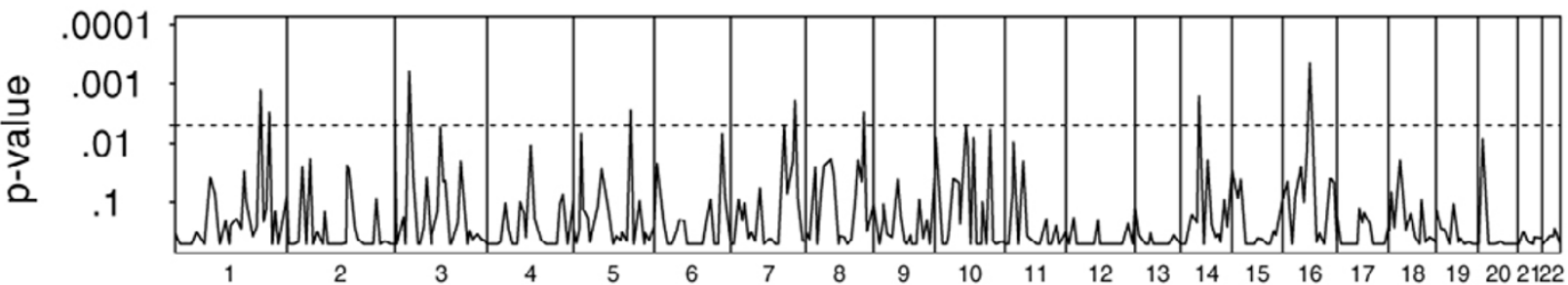

Chromosome

Figure I

p-values for the three statistics throughout the genome Bivariate score statistic $(A)$, univariate score statistic for adjusted cholesterol level (B), and univariate score statistic for adjusted triglyceride level (C). 
Table 2: Summary of markers that are significant at significance level 0.005

\begin{tabular}{|c|c|c|c|c|c|}
\hline \multirow[t]{2}{*}{ Chr. } & \multirow[t]{2}{*}{ Marker } & \multirow[t]{2}{*}{ p-telomere (cM) } & \multirow{2}{*}{$\begin{array}{l}\text { Bivariate Score } \\
\text { statistic }\end{array}$} & \multicolumn{2}{|c|}{ Univariate score statistic } \\
\hline & & & & Cholesterol & Triglyceride \\
\hline I & Gata48B0 I & 212 & $11.029(.0004)$ & & $9.141(.0013)$ \\
\hline I & GATA87F04 & 233 & $8.618(.0017)$ & & $7.602(.0029)$ \\
\hline 3 & 3PTEL25 & 1 & & & $8.358(.0019)$ \\
\hline 3 & $036 y b 8$ & 37 & & & $10.445(.0006)$ \\
\hline 3 & GATAI $28 \mathrm{C} 02$ & 112 & $6.799(.0046)$ & & \\
\hline 4 & GATA24H0I & 78 & & $6.670(.0049)$ & \\
\hline 4 & ATA2A03 & 93 & & $7.665(.0028)$ & \\
\hline 4 & GATA2FII & 105 & $7.534(.0030)$ & & \\
\hline 4 & ATA26B08 & 130 & & $7.159(.0037)$ & \\
\hline 5 & GATA2A04 & 19 & $7.119(.0038)$ & & \\
\hline 5 & GATA2H09 & 139 & & & $7.682(.0028)$ \\
\hline 6 & $242 z g 5$ & 166 & $7.776(.0026)$ & & \\
\hline 7 & GGAA6D03 & 128 & $7.036(.0040)$ & & \\
\hline 7 & GATAII 2F07 & 155 & $8.274(.0020)$ & & $8.410(.0019)$ \\
\hline 8 & GATA2ICI 2 & 140 & $10.474(.0006)$ & & $7.603(.0029)$ \\
\hline 12 & GATA49DI 2 & 18 & & $7.695(.0028)$ & \\
\hline 12 & GATA3F02 & 81 & & $9.674(.0009)$ & \\
\hline 14 & GATA4B04 & 44 & & & $8.648(.0016)$ \\
\hline 16 & ATA55AII & 64 & $13.489(.000 \mathrm{I})$ & & $11.049(.0004)$ \\
\hline 21 & GGAA3C07 & 13 & & $7.043(.0040)$ & \\
\hline
\end{tabular}




\section{Acknowledgments}

We thank three anonymous reviewers for their constructive comments. This work is supported in part by a clinical research center grant PO-DC02748 from the National Institute on Deafness and Other Communication Disorders (PI: Dr. J. Bruce Tomblin; Investigators include XZ) and National Institute of Mental Health grant R0I-5284I (PI: Dr. Veronica Vieland; Investigators include $\mathrm{KW}$ ).

\section{References}

I. Wang K: Mapping quantitative trait loci using multiple phenotypes in general pedigrees. Hum Hered 2003 in press.

2. Amos Cl, Elston RC, Wilson RF, Bailey-Wilson JE: A more powerful robust sib-pair test of linkage for quantitative traits. Genet Epidemiol 1989, 6:435-449.

3. Wang K, Huang J: A score-statistic approach for mapping quantitative-trait loci with sibships of arbitrary size. Am J Hum Genet 2002, 70:412-424

4. Sham PC, Purcell S: Equivalence between Haseman-Elston and variance-components linkage analyses for sib pairs. Am J Hum Genet 200I, 68:1527-I532.

5. Putter H, Sandkuijl LA, van Houwelingen JC: Score test for detecting linkage to quantitative traits. Genet Epidemiol 2002, 22:345-355.

6. Shearman AM, Ordovas JM, Cupples LA, Schaefer EJ, Harmon MD, Shao Y, Keen JD, DeStefano AL, Joost O, Wilson PWF, Housman DE, Myers RH: Evidence for a gene influencing the TG/HDL-C ratio on chromosome 7q32.3-qter: a genome-wide scan in the Framingham Study. Hum Mol Genet 2000, 9:13 I5-1320.

7. Jiang $C$, Zeng ZB: Multiple trait analysis of genetic mapping for quantitative trait loci. Genetics 1995, I40: I I II-I I 27.

8. Allison DB, Thiel B, Jean PS, Elston RC, Intante MC, Schork NJ: Multiple phenotype modeling in gene-mapping studies of quantitative traits: power advantages. Am J Hum Genet 1998, 63:1190-1201.

9. Amos $\mathrm{Cl}$, de Andrade $\mathrm{M}$, Zhu DK: Comparison of multivariate tests for genetic linkage. Hum Hered 200 I, 5 I : I33- I 44.

Publish with Bio Med Central and every scientist can read your work free of charge

"BioMed Central will be the most significant development for disseminating the results of biomedical research in our lifetime. "

Sir Paul Nurse, Cancer Research UK

Your research papers will be:

- available free of charge to the entire biomedical community

- peer reviewed and published immediately upon acceptance

- cited in PubMed and archived on PubMed Central

- yours - you keep the copyright 